\title{
Primitive and Schedule-Based Sensor Networks MAC Protocol: A Survey
}

\author{
Pallavi.M ${ }^{1}$ \\ ${ }^{1}$ Research Scholar, Department of ECE \\ Dayananda Sagar College of Engineering, Bangalore, Karnataka, India \\ pallaviait@gmail.com \\ Dr. V.Anitha ${ }^{2}$ \\ ${ }^{2}$ Professor, Department of ECE \\ Dayananda Sagar College of Engineering, Bangalore, Karnataka, India \\ anithavijaya@gmail.com
}

\begin{abstract}
Wireless Sensor Networks (WSNs) are endearing to researchers in view of their outspread application capacity in the multifarious domain such as object detection and tracking, industrial automation, environmental monitoring, smart home, and tactical system [1]. Typically, a WSN comprises of enormous low-cost and small sensor nodes which are deployed in an intended region to acquire data of interest. However, poor sensing range of a node evolves compact network, hence developing a dynamic Medium-Access Control (MAC) protocol is predominant. So far, a great number of MAC protocols have been presented with different ideas for wireless sensor networks. Initially, researchers' prime consideration was energy efficiency. In the recent past, researchers have given priority to design a protocol that supports multitasking while being adaptive to traffic loads. In this paper, an attempt has been made to survey the numerous Primitive and Schedule-Based MAC protocols. At first, the properties of wireless sensors paramount for the design of MAC layer protocols are summarized. Then, the Primitive MAC protocols and Scheduled-Based MAC protocols are discussed, and finally, their advantages and disadvantages are emphasized.
\end{abstract}

Keywords - Energy Efficiency, FDMA, Medium Access Control (MAC), TDMA, Wireless Sensor Networks.

\section{INTRODUCTION TO WSNS MAC}

Great progress in technology has emanated the evolution of small, inexpensive sensor nodes which are embedded with various sections like sensing, processing, and communication. The vast application scope has been encouraged by sensor nodes through wireless sensor networking [2][3]. The pivotal aspect of WSN protocol design is to enhance energy efficiency, considering the sensor nodes which are required to work independently with smaller batteries while boosting sensor node lifespan. Since each node comprises of a transceiver that communicates with other nodes, battery span plays an important role in node health. Since the WSN are expected to work efficiently with minimal human intervention, it is highly desired to design a protocol that produces very little bottlenecks. Medium access control is a subsection of the data link layer which is the most widely considered layer for the accurate functioning of any communication system. The fundamental work of MAC is to organize transmission and reception over a medium common to various nodes. Since WSN comprises of a large number of nodes communicating with one another, it is essential to have an accurate MAC protocol to increase the WSN efficiency. This has caused a widespread research on designing an efficient MAC protocol [4]. An energy efficient MAC protocol enhances the lifespan of a sensor network to a larger extent by regulating the transceiver, which is a crucial energy consuming component. In addition, it mitigates the collisions and enhances the throughput. There have been a prodigious number of investigations on the design and implementation of MAC protocols in WSNs. Therefore, it is essential to conduct an effective survey on WSN MAC protocols to encapsulate the various design and implementation aspects.

Various MAC protocol design depends on medium access schemes are discussed in [5]. Later, in [6] a research work was conducted to inspect the strengths and weaknesses of various MAC protocols. Further extensive work in [7] categorized WSN MAC protocol into four classes, which are mainly based on homogeneity and nonhomogeneity. Primitive design of MAC protocols have given more importance to enhancing the lifetime of sensor nodes by sacrificing throughput and delay. However, with advancements in technology new protocols have been designed to encourage multitask and efficient delivery. In this paper, besides classifying the protocols, some ideas based on essential development flow like objectives that motivated the protocol design, their disadvantages, and bottlenecks in available protocols, and how present and developing technology will impact future research are discussed. 


\section{MEDIUM ACCESS MECHANISM}

Basically, the Radio frequency (RF) spectrum is segregated into various bands, and these bands are assigned to numerous systems based on the requirements. The assigned bands are further divided into channels using different methods like frequency division multiple access (FDMA), time division multiple access (TDMA) and spread spectrum techniques such as code division multiple access (CDMA) etc. The assigning of individual channels to various devices may be fixed or dynamic. Practically, when a large number of working systems are appended to a wireless medium, it is almost unfeasible to assign an individual channel to each device. In "OSI model" MAC is a sub-layer of the Data Link layer. The main function of MAC protocols is to regulate the channel assignment.

\section{A. Main Sources of Energy}

Basically, the main sources of energy waste in a wireless sensor network include Fame collisions, Message overhearing, Idle listening, and Protocol overhead.

i. Frame collisions: Frame collision occurs when a transmitted data imbricate in time with other data. Due to a collision, the transmitter receives an acknowledgement (ACK) from the receiver which reveals the data transmission failure. Thus, transmitter institutes a new link with the receiver to retransmit the data packet to increase the successfully received probability. But, the establishment of new links with the receiver and retransmission of data packets dissipate extra energy. The credibility of collision can be reduced by employing back-off algorithms or scheduling protocols.

ii. Message overhearing: Overhearing occurs when a node receives a message which is destined to another node. In non-energy efficient methods, these messages will simply be discarded to maximize the throughput and minimize the latency. But, in energy-efficient methods, early rejection and Network Allocation Vector (NAV) sleep are the best solutions to mitigate overhearing.

iii. Idle listening: Here, a node is active for a long duration without any transmission or reception which causes high energy dissipation. An attempt has been made in Contention-based WSN MAC protocols to mitigate the idle listening by initiating transmission only during fixed or predetermined slots during which only those nodes with data for transmission or reception will be active. Once the transmission process is complete it allows nodes to go back to sleep until next transmission.

iv. Protocol overhead: Collision and exchange of a large number of control messages are the main causes of overhead. If a data packet collides with another data packet, the transmitter has to retransmit the packet again which consumes extra energy and causes overhead. To mitigate the overhead, the designer should minimize the exchange of control messages.

\section{PRIMITIVE OR TRADITIONAL MAC PROTOCOLS (Canonical Solution for Sources of energy)}

In Carrier Sense Multiple Access /Collisions Avoidance (CSMA/CA), before establishing the communication with the intended receiver, a transmitter sends a tiny Request to Send (RTS) packet to the receiver. When the intended receiver receives an RTS packet, it acknowledges by sending a (CTS) packet. The function of CTS packet is to preserve the channels surrounding the receiver to mitigate any collision with the active transmission. Disadvantages: i. It mitigates the performance because collision probability and RTS packets are in same order. ii. The utility of RTS/CTS enhances the energy dissipation. iii. RTS/CTS packets encourage only unicast transmission.

Multiple Access Collision Avoidance (MACA) [8] is an advancement for CSMA/CA. It appends a random back-off period before sending the RTS packet to mitigate collisions occurs from synchronized forwarding by multiple neighbors. Usually, the backoff period is selected based on a uniform distribution which is not the ideal choice.

Adaptive Rate Control (CSMA/ARC) [9][10] MAC protocol is an enhancement for the traditional CSMA/CA. It mitigates the overhead in CSMA/CA technique by eliminating RTS/CTS packets and providing a back-off that will adapt based on the application revolution. Hence, this technique limits both back-off time and collision. It assures fairness between forwarded and the generated traffic loads. Here, the data packet will evade extensive use of ACK packets by considering data packets will successfully reach the receiver if the upstream node forwards the data packet to next nodes fairly. Hence, it further mitigates the overhead.

Power-Aware Multi-Access with Signaling (PAMAS) [11] is proposed for ad-hoc network with dense traffic loads which is distinct from sensor networks. In the ad-hoc network, functions like frequent communication and thus energy conservation are common, but sensor networks have practically low traffic load hence RTS/CTS are usually excluded.

In IEEE 802.11 Power Save Mode (PSM) [10] nodes switch to sleep mode to mitigate energy dissipation. PSM is broadly classified into two types based on the availability of access point: PSM with a Basic service set (BSS) and PSM with an Independent BSS (IBSS). 
i. $\quad$ PSM with a BSS (with the access point): Initially, a node transmits the data packet to notify the access point; if it receives an acknowledgement from the AP then it starts its PSM operation. In PSM, nodes remain predominantly idle for a long time but become periodically dynamic to receive the signal from the AP. The AP cannot send data to a node in PSM mode, because of the long idle period. Hence, it buffers all data routed to nodes that are in PSM mode. The signals transmitted by the AP contain Traffic Indication Map (TIM) comprising pending packet guidance. Therefore, when a node receives a signal, it is able to search for pending data corresponding to it. Here, nodes have two choices to access pending packets based on whether the packet is unicast or broadcast. For unicast packets, the node decides when to access it, and if it is ready to receive the data it acknowledges to AP through the poll packet. For the broadcast packets, AP decides when to transmit and it is the choice let for the node whether to access the data or not.

ii. $\quad$ PSM with IBSS (without the access point): In this approach, nodes are synchronized with the regular broadcasting of signal packets. Initially, Announcement Traffic Indication Message (ATIM) is broadcasted when all nodes are active. Based on this message, transmitter node will verify whether the destination node is in power save mode, before sending the packet. If it is in PSM, during ATIM window where all nodes are awake it announces the packet to be transmitted. After listening to the announcement, destination node sends an acknowledgment to the sender and it continues to be active to collect the data packet.

TABLE I

Summary of Primitive or Traditional MAC.

\begin{tabular}{|c|c|c|c|c|}
\hline $\begin{array}{l}\text { Sl. } \\
\text { No. }\end{array}$ & Protocol & Basic Technique & Advantage & Disadvantage \\
\hline \multirow[t]{2}{*}{1} & \multirow[t]{2}{*}{ CSMA/CA } & \multirow{2}{*}{$\begin{array}{l}\text { Communication } \\
\text { RTS/CTS packets }\end{array}$} & \multirow[t]{2}{*}{ Collision avoidance } & High energy dissipation \\
\hline & & & & $\begin{array}{l}\text { Support only unicast } \\
\text { transmission }\end{array}$ \\
\hline 2 & MACA & $\begin{array}{l}\text { RTS/CTS packet+ Random } \\
\text { back-off period before RTS }\end{array}$ & Collision avoidance & $\begin{array}{l}\text { Additional } \\
\text { dissipation due to back-off } \\
\text { period }\end{array}$ \\
\hline \multirow[t]{2}{*}{3} & \multirow[t]{2}{*}{ CSMA/ARC } & \multirow[t]{2}{*}{ Only Back-off period } & \multirow[t]{2}{*}{ Mitigates overhead } & Complex \\
\hline & & & & Introduces collision \\
\hline 4 & PAMAC & $\begin{array}{l}\text { Communication } \\
\text { RTS/CTS packets }\end{array}$ & Mitigates overhearing & Energy dissipation \\
\hline \multirow[t]{2}{*}{5} & \multirow[t]{2}{*}{$\begin{array}{l}\text { IEEE } \\
\text { PSM }\end{array}$} & \multirow{2}{*}{$\begin{array}{l}\text { PSM with a BSS (Basic } \\
\text { Service Set) and PSM with an } \\
\text { IBSS (Independent BSS) }\end{array}$} & \multirow[t]{2}{*}{ Mitigates idle listening } & $\begin{array}{l}\text { This will not support multi- } \\
\text { hop network }\end{array}$ \\
\hline & & & & Latency is high \\
\hline
\end{tabular}

\section{SCHEDULED-BASED MAC PROTOCOLS}

A. Basic Technique: In schedule-based MAC, nodes only wake up and listen to the channel in allotted slots and then go back to sleep in other slots. This mechanism requires the information on network topology and channels to communicate with other nodes without any interference by another transmission. The protocols with Scheduled mechanism are discussed below:

i. $\quad$ Time Synchronized Mesh Protocol (TSMP) [12] is a medium access and networking protocol proposed for the Wireless HART standard in industrial automation. TSMP yields reliable data transfer by integrating time, frequency, and routing diversity. Here, each node shares the same sense of time, accurate to $<1 \mathrm{~ms}$, which enables time-diverseness employing discrete transmissions to arise during the non-overlapping span and hence preserve energy by evading collisions. Based on when transmission could appear, nodes on both sides of a radio link can mitigate their "on time", and cost of conserving this synch is less in contrast to asynchronous protocols [13][14]. TSMP communication employs no additional preamble above physical-layer (PHY) demands and low cost of idle listening is accomplished by employing $2 \mathrm{~ms}$ guard duration. For identical node pair, packet delivery rates on distinct channels can broadly fluctuate, the alike single channel over time [15]. By appending channel hopping in addition to DSSS (Direct Sequence Spread Spectrum), TSMP provides frequency-diverse benefits contrast to a single-channel solution: a. It linearly improves network bandwidth in various channels. b. It improves reliability by mitigating terminal loss. c. More channel usage improves effective radio range due to frequency dependent multipath fading.

ii. $\quad$ Power efficient and delay aware medium access control protocol for sensor networks (PEDAMACS ) [16] is a TDMA based MAC protocol. Initially, it collects the topology information by activating the access point. Based on the topology information, it constructs a periodic schedule. This schedule has the complete information about when each node should be active, when it has to transmit or receive data, and when it has to return to sleep state etc. When network topology encounters any deviation, it activates the access point again to collect the new information. This protocol uses polynomial-time scheduling algorithm instead of an optimum 
scheduling algorithm to achieve guaranteed delay. This yields better efficiency than random access protocols by allowing each node to sleep when they are idle or free.

iii. BitMAC [17] protocol is a deterministic, collision-free, and robust protocol desirable for a compact WSNs. Basically, BitMAC will operate as a spanning tree, in which tree root is the sink. This tree comprises a several interdependent star network, where each star network is constructed by the internal node and its direct children. Each node acts as an access point for its direct children. The TDMA technique is employed for each star network to evade communication interference between the children and the parent. Here, Time slots are assigned to nodes only when it is essential. In BitMAC technique, to evade the interference between neighboring stars, a distributed graph coloring algorithm is employed. The operation of BitMAC and IEEE802.11 is practically alike.

iv. G-MAC [18] is an adaptive WSN MAC protocol, which achieves a novel cluster-centric standard for the successful allocation of cluster energy resources to expand the energy efficiency. The cluster centric management tenders considerable energy saving by trading between the benefits of contention and contentionfree protocols. Both the contention based and contention free schemes tender same network scalability but the contention free scheme provides better network stability for dense traffic loads. The TDMA frame of G-MAC comprises three sessions: the collection phase, the traffic indication phase, and the distribution phase. Initially, in the collection phase, the cluster coordinator, called the gateway, gathers two types of network traffic requests: intra-network (local) and inter-network (non-local) traffic. Intra-network traffic presents data transmitted between nodes in the same cluster. Here, the transmitter sends an FRTS message to secure a slot in the contention free period. But in inter-network traffic presents data to be transmitted or received by the gateway from outside node. After completing the transaction, the gateway broadcasts the traffic information to all nodes and then nodes are authorized to sleep. Secondly, in distribution period, every node wakes up to collect the gateway traffic indication message (GTIM) which broadcast the scheduled information. The nodes which are scheduled to send or receive the data sleeps until the exchange time, and become dynamic during its allotted period and later return to sleep. The nodes which are not scheduled will be inactive for the entire distribution period. The limitation of G-MAC is the large overhead, especially in the collection phase which increases the mean energy consumption per node. Another drawback is the demands for more gateways due to direct communication of nodes with gateways.

v. Self-Organizing Medium Access Control for Sensor Networks (SMACS) [19] protocol, nodes are independent to construct a communication framework without a master node as in centralized scheduling mechanism between neighboring nodes by specifying transmission and reception slots. Here, Channel accessing procedure employs the combination of TDMA and FDMA or CDMA. Basically, it employs two working phases: neighbor discovery and channel assignment. In the neighbor discovery phase, a node is active for a finite duration waiting for invitation packets to discover its neighbor; if it does not encounter any packet then it begins to invite others by transmitting an invitation packet. In the channel assignment phase, they create transmission-reception slots after establishing a connection between nodes. In SMACS, to mitigate collisions, each node link selects random frequency/channel from a pool, and to conserve energy nodes sleep randomly.

vi. The traffic-adaptive medium access protocol (TRAMA) [20]is TDMA based energy-efficient protocol in wireless sensor networks. TRAMA employs traffic-based scheduling to evade wasting slots when nodes do not have information to broadcast and redirect nodes to a low-power standby radio mode when they are not deliberated receivers of traffic. This protocol utilizes a contention period for two-hop topology construction and a contention free period for data exchange. The distributed election algorithm in TRAMA eradicates the hiddenterminal issues and hence guarantees that all nodes in the one-hop neighborhood of the transmitter will receive information with no collision. TRAMA comprises of three main sections:

a. The neighbor protocol - For gathering the information of neighboring nodes.

b. The schedule exchange protocol- For trading the two-hop neighbor data and their schedule.

c. The adaptive election algorithm- Choosing the transmitting and receiving nodes for the current time slot based on the neighborhood and schedule information. Simulation outcome manifest that, since node can sleep up to $87 \%$ it is capable of yielding significant energy saving, and also achieves higher throughput in contrast to contention-based protocol by evading collision due to hidden terminals.

vii. $\quad \mu$ MAC [21] Protocol operates sequentially between a contention and a contention-free phases. Contention phase is employed to establish a network topology and to initialize communication sub-channels and contention free period is employed to send data packets between the nodes. The protocol operation is divided into three stages: In the first stage, the base-station shows an interest by requesting for network data. In the second stage, if a sensor is capable to generate the requested data from the network then it provides a reserve command to $\mu \mathrm{MAC}$. Finally, in the third stage, after requesting the reservation of a sensor report sub-channel, the data produced by sensor will report towards the base-station. Reports are transferred between nodes through calls to the report command.

viii. Energy efficient MAC (EMACs) [22]is a fully distributed and self-organizing TDMA protocol which is designed to minimize energy consumption, while minimizing delay and loss of data throughput. The 
working of EMAC is divided into three stages: active, passive and dormant stage. In an active stage, nodes will be busy in transmitting messages to the destination and receiving data from passive nodes. In Passive stage, nodes will trace only one active node thus preserving the energy. In dormant stage, nodes place themselves in a sleep state for finite time interval. In TDMA, time is divided into frames and each frame is divided into slots. Each slot has three parts: Communication Request (CR), Topology Control (TC), and DATA. In CR section, active node listens to incoming passive node request. In TC section, it broadcasts a small control message which comprises a possible ACK to the request, control and synchronization information. Here, nodes have the knowledge of their local neighborhood since they listen to their neighbor TCs. Finally, the data section is employed for the actual data transfer from higher network protocol layers. The drawback of EMACs is, the active nodes are active in the entire slot thus leading to more energy dissipation. Through simulation performance, the EMAC protocol is capable of enhancing the network lifetime by $30 \%$ to $55 \%$ contrast to SMAC.

ix. Positioning-enabled medium-access control (PMAC) [23] protocol is proposed for ultra-wideband sensor networks. It is a simple, TDMA based protocol which encourages both localization and energy efficiency. Basically, it does not depend on base stations or a central access point but it endorses a selforganizing, dynamic topology hence it is suitable for wireless ad-hoc networks. Here, each slot is divided into three sections which is similar to EMAC: dynamic, passive and sleep section. In dynamic section, nodes will establish a communication path which encourages data relying and broadcasting to a specific destination by acquiring data from the passive nodes. In the passive section, it conserves energy by tracing only dynamic nodes. Data position information can be evaluated at any time slot because there is a sub-slot within a time slot for updating the position. The basic idea of this protocol is to enable short omnidirectional data transmission between the UWB nodes and upper network layers which transfer local topology information to the higher network layers. Finally, this protocol also enables all nodes to have information on their physical area and operating position.

x. Power Aware Clustered TDMA (PACT) [24] is an innovative energy-efficient TDMA protocol. PACT utilizes both space and time to accomplish power savings. Based on Spatial clustered hierarchical structure, network layer protocols are enabled to appoint only a set of nodes for routing packets from a densely deployed sensor network. In addition, the clustering algorithm adjusts to the energy level of the nodes in the network and switches the role of cluster heads and gateways accordingly. PACT employs TDMA based scheduling to turn off radio circuitry during idle phase to conserve power in the case of time domain. Thus, all nodes adapts its duty cycles to higher layer data traffic and mitigates the necessity of keeping the radio constantly on as in a random access scheme (e.g., IEEE802.11). Compared to contention-based MAC protocol such as IEEE 802.11, it greatly reduces the idle energy dissipation by powering down the radio during idle slots. Compared to IEEE 802.11, PACT enhances the network lifetime by more than 5 times. It adapts energy utilization to user traffic, and also elongate the network lifetime dense topologies with large traffic load which is achieved through passive clustering. PACT is the prime TDMA protocol that employs clustering to gain benefits of dense topology to conserve energy and elongate the lifetime of network. PACT can smartly enhance the sensor network functional time furthermore with rechargeable batteries.

xi. Bit-map-assisted (BMA) [25] MAC protocol is a large-scale cluster-oriented WSNs. It is designed predominantly for event-driven approaches, where source node sends the data packet to the cluster head only for essential events. Its prime objective is to mitigate the energy dissipation by idle listening and collision and to support low-latency operation. The basic operation of BMA is divided into two sections: cluster set-up and a steady-state section. In set-up section, based on the energy level, nodes are allowed to compete for cluster head, and selected nodes are declared as a new cluster head. Next, each non-cluster head amalgamate with the suitable clusters based on the energy level. Finally, the system pervade to steady-state section after constructing a cluster. In a steady-state phase, each session is divided into three periods: a contention period, a data transmission period and an idle period. The contention period supports a TDMA schedule where a particular slot is allotted to each node. Nodes with data packet are called source nodes, which transmits a control message in its allotted slot otherwise the allotted slot is empty. The system enters directly to idle period if none of the non-cluster head nodes have data to transmit. In transmission period, source nodes will become active during its scheduled slot to transmit data packet to the cluster head, after transmitting, it turns off its radio. Finally, in idle period, all source and non-source nodes turns off their radios till the next session.

xii. Mobility adaptive medium access control (MMAC) [26] protocol employs similar design principle as TRAMA protocol. This regales both weak mobility (due to topology adjustment, node interconnection) and strong mobility (synchronous node interconnection and physical adaptability of nodes). It establish flexible mobility frame period which facilitate each protocol with dynamic adaptability over the mobility patterns, allowing it as a desirable technique to work in both high and low sensor environments. This method mitigates the collision by employing scheduled-based approach. MMAC assumes that the sensor nodes are aware of their location and hence this location information is used to estimate/predict mobility patterns. The major issue in MMAC is the demand for position information, which is practically inconvenient and dissipates excess energy. 
xiii. Flexible MAC (FlexiMAC) [27] protocol is a TDMA based, self-healing protocol developed for regular data clustering applications. It yields guaranteed end-to-end delay for packet delivery, better throughput, tolerable data access, and vigorous self-healing while preserving energy and memory constraints. FlexiMAC is synchronized and employs flexible slot structure which facilitates a node to develop, alter, or enhance their allotted slot numbers during execution depends on the local information. Hence, this technique is highly fault tolerant and energy efficient. In FlexiMAC, slot 1 is reserved for Fault-Tolerant Slot (FTS) for controlling the network mobility; hence slot allotment for nodes starts from 2. New nodes utilize FTS to request for communication slots. Here, time slot allocation procedure follows two steps: data gathering slot allocation and multi-functional slot (MFS) allocation. Data gathering slots are employed to establish a communication path from nodes to sink and MFS is used for local time synchronization and local restoration. It further mitigates energy dissipation by employing optimal node transmission power. It implements communication slot reuse approach hence FlexiMAC is scalable for dense network and it mitigates buffering by employing depth-firstsearch-schedule. Here, it is not needed to define the number of slots required for the network in advance and nodes can join or leave the network at any time.

xiv. Off MAC (O-MAC) [28] is a TDMA protocol which employs receiver based scheduling to enhance the energy efficiency by the orders of magnitude. The working principle of O-MAC is similar to Crankshaft. Basically, power dissipation is dominant in receiver nodes in contrast to sender nodes; therefore O-MAC is developed to mitigate energy dissipation in receiver section. It employs two receiver based scheduling techniques: centralized deterministic scheduling (Staggered On) and decentralized pseudo-random scheduling (Pseudorandom Staggered On). In Staggered On, all the receivers are organized to wake up to evade the interference between the receivers; therefore a receiver with overlapping interference region will not be active at the same time. In this method, there is guaranteed collision avoidance. Pseudorandom Staggered On mitigates the problems like implementing and regulating a global schedule in the case of Staggered On scheme by allowing each node to wake up independently with finite probability.

$x v$. The Crankshaft [29] is a hybrid MAC protocol like SCP, proposed to regulate the prime source of inefficiency in crowded network by utilizing node synchronization and offset wake up. The fundamental principle is, in a frame, nodes are active barely for a fixed offset to receive messages. Crankshaft splits time into frames, and frame is divided into broadcast and unicast slots. It is an essential for each node to listen for one unicast slot and to all broadcast slots for every frame based on its MAC address. Here, to broadcast a message from sender to destined node, a sender node must wait and compete for destined node's unicast slot using contention idea, since various nodes might desire to transmit a data in a specific slot. However, in crankshaft protocol, the unoccupied slots mitigate the overall throughput at low traffic load.

xvi. PicoRadio [30] sensor network, an exploration attempt of the Berkeley Wireless Research Center. It comprises of small, lightweight, low-cost network elements called Pico-Nodes. To accomplish maximal network lifetime, it is essential to mitigate the energy consumption from each layers. The pivotal design objective of PicoRadio is an ultra-power sensor network with inexpensive nodes $(<1 \$)$, which are tiny $(<$ one cubic centimeters) and are battery operated. The hard-ware and the software design are desired for a level of power dissipation below 100 microwatts, where as a power dissipation in Bluetooth radio is more than 100 milliwatts. The PicoRadio protocol stack is developed with the premise that each node has the similar capabilities (battery, processor power) and every protocol perform in a totally decentralized mode. Each Pico-Node listens to a common control channel and transmits a channel assignment packet to educate its neighbors about its channel. It continues to trace its entire one and two-hop neighbors channels to evade selecting an overlapping channel with them. During channel configuration phase, nodes perform the following mechanisms: an Individual node collects information of used channels of its neighbors when it is awakened. It then employs another unused channel from the channel pool and transmits selected channel and neighbor's channel. If a dispute happens, the node that first diagnoses the dispute switches to another unused channel.

xvii. framelet MAC (f-MAC) [31] protocol is proposed to yield assured message delay and channel throughput without the need for time synchronization between the nodes. In this approach, a single data is broadcasted many a times using small and fixed sized data packets, where each data packet is called a framelet. Data transmission of same data reduces the available bandwidth. Since each interfering nodes functions at a discrete frequency, the probability of collision is less. Here, nodes will be active for most of the time to check for the channel and to access the framelet hence energy efficiency is less.

xviii. Lightweight MAC (LMAC) [32] protocol is a self-regulating TDMA scheme that group's time into frames carrying the number of slots. Each node possess one slot in which it broadcasts a header to imprint its occupancy, conceivably followed by a data payload either directed to a definite receiver or to all nodes in range. Thus, a node must listen in all slots other than its own to monitor for incoming data. The header contains a list of all invaded slots in the owner's one-hop neighborhood to permit for collision-free broadcasts and spatial reuse of slots. After combining the occupancy data of its neighbors, a fresh node binding the network appoints a free transmission slot within its two-hop neighborhood. This dispersed free slot selection mechanism enables the elective ACK messages. Multichannel LMAC amplifies the LMAC by appending multi-channel support. 
The objective of the protocol is to mitigate the number of transceiver switches, to establish the sleep interval for sensor nodes flexible to the number of data traffic and to restrict the difficult of execution.

xix. Multichannel LMAC (MC-LMAC) [33] is proposed to upgrade the performance of LMAC. The performance of single-channel MAC curtail in densely deployed network due to the greater demand for the limited bandwidth. Traditional LMAC employs TDMA scheduling, where different nodes are allotted to different slots in a distributed fashion. In highly dense network regions, all slots are engaged and fresh nodes may end up without any slot for communication. To evade LMAC limitation, multichannel LMAC enables the nodes to initiate fresh on-demand channels, if network enters a saturation density limit. This method is comprised of two working steps: In the first step, nodes follow the single-channel LMAC rules to choose the timeslot. In the second, the nodes which are unable to fetch a timeslot in the first step gathers its free neighbor nodes which are agreed to listen to them in a specified frequency channel and timeslot. Multichannel LMAC needs only one half-duplex transceiver per node for the communication.

xx. Adaptive, Information-centric and Lightweight MAC (AI-LMAC) [34] is a TDMA based protocol, which is adroit to adopt its operation based on the application requirements. In this method, data management framework is inhabited on each node. Initially, Data Distribution Tables (DDTs) are established to minimize the kind of expected data traffic based on the incoming queries and distribution of generated data packets. If a node receives a query, it checks its DDTs to find how many of its children are going to acknowledge to the specific query. Each node conserves an independent set of DDTs to maintain information regarding the type and availability of sensors within its child node. After receiving the measurements from the child nodes, the parent node upgrade the DDTs. AI-LMAC is application-oriented protocol; it reduces latency and regulates the fairness issue in a better way by employing two-dimensional fairness mechanism.

xxi. Multi-frequency Media access control for wireless Sensor Network (MMSN) [35] protocol is the elemental multi frequency MAC protocol, predominantly developed for WSNs. In MMSN every device acquires a single radio transceiver with compact MAC layer packet size. It encompassed with hundreds of simple devices distributed over an enormous geographic area in an ad hoc fashion. The MMSN protocol comprises two features: frequency assignment and media access. The frequency assignment is employed to allocate distinct frequencies if sufficient frequencies are available, or uniformly assign available frequencies if there are more neighbors than available frequencies. It enables users to adopt 1 of 4 available frequency allocation procedures. It presents the outstanding potential to employ parallel transmissions among neighboring nodes. MMSN also accomplish increased energy efficiency as well as exhibits the propensity to perform against radio interference and the patience to a wide range of measured time synchronization errors for multiple physical frequencies.

xxii. Y-MAC [36] is a TDMA scheduling multi-channel MAC protocol proposed to achieve better performance and energy efficiency under diverse traffic conditions. Here, time is divided into fixed length frames, and each frame comprised with unicast period and broadcast period. In Y-MAC, nodes wake up at discrete time. Initially, during broadcast period, nodes will become active to trade broadcast messages. When a node finds, there are no broadcast messages directed to it, it goes back to sleep by turning off its radio. More number of time slots enables large number of node allocation but delivery latency enhances due to extended frame duration length. Hence, it is essential to regulate the number of required time slot for satisfactory performance. One more possibility to raise the number of time slot is by employing the multiple channels. The practice of multiple channels will enhance the MAC protocol performance with low energy dissipation. In YMAC, light-weight channel hopping technique enables simultaneous communication of multiple nodes on multiple channels, and if there are any pending data packets for the receiver then sensor nodes drive to next radio channel. This technique enhances the network throughput and mitigates packet delivery latency at the same time.

xxiii. Practical multichannel MAC (PMMAC) [37] employs an idea of allocating home frequency to all nodes to amplify network throughput. Here each divergent frequency accessible to the network is called a channel. Initially, every node begins to fill the same channel, when it becomes overloaded, remaining nodes move to other channels to circulate the communication load across non-interfering frequencies. Emigration to other home frequency does not demand connectivity loss from nodes attached to previous home frequency. Instead, it is authorized to broadcast message to other nodes. Inter-node transmission is done by temporarily switching to the destination's channel. Although nodes do not need to be synchronized, it needs to send their status neighbors frequently.

xxiv. Self-Stabilizing TDMA (SS-TDMA) [38] protocol is proposed for grid-based sensor network. Generally, there are three communication patterns: broadcast convergecast and local gossip which occur frequently in the grid-based systems. In SS-TDMA, sensors are allowed to enter and continued to be in power save mode as long as the other sensors remain connected. The sleeping sensors will wake up systematically, wait for diffusion message from its neighbor and finally goes back to sleep. This idea encourages better energy conservation and clock synchronization with the neighbor nodes. In this method, it is assumed that sensors are organized in a grid; hence sensors are aware of its geometric position (location in the network). Each data transmitted by a 
sensor includes the location. Therefore, sensors are able to discover the position, direction and distance of data from it. SS-TDMA design can also be extended for non-grid topologies, mobile sensor nodes and failed sensors. Hence, it is considered as an efficient method to handle regular issues: failed sensors, sleeping sensors, unidirectional links, and unreliable links.

xxv. Randomized MAC (RMAC) [39] is a simple, adaptive TDMA based protocol for heterogeneous sensor networks. This protocol is designed for serving communication between the higher and lowers level nodes. The working of RMAC is divided into three phases: $i$. in the first phase host estimates the number of data packets using Maximum-Likelihood estimation technique, based on this information it decides and announces number of fixed-size mini-slots. The nodes with data packet to transmit select mini-slots uniformly, and request for the reservation. Nodes are allowed to pre-reserve a slot in the current round by sending a request in the previous round. Here, host invests the mini-slots to find the collision. ii. In second phase, host broadcast the number and specification of nodes which are reserved the mini-slots. iii. In the third phase, allotted nodes will send their data packets. Hence, collision is successfully mitigated in RMAC. The nodes which are unable to get the minislots at the current round can attempt once again in the next round. R-MAC is an energy efficient MAC and results better fairness.

TABLE II

Summary of Scheduled-Based MAC Protocol

\begin{tabular}{|c|c|c|c|c|}
\hline $\begin{array}{l}\text { Sl. } \\
\text { No. }\end{array}$ & Protocol & Basic Technique & Advantage & Disadvantage \\
\hline \multirow[t]{2}{*}{1} & \multirow[t]{2}{*}{ TSMP } & \multirow[t]{2}{*}{ Synchronous TDMA Scheme } & \multirow[t]{2}{*}{ High Robustness } & Complex to implement \\
\hline & & & & High cost \\
\hline 2 & PEDAMACS & $\begin{array}{l}\text { Centralized Scheduling TDMA } \\
\text { scheme }\end{array}$ & Mitigates Collision & $\begin{array}{l}\text { Supports only convergecast } \\
\text { traffic pattern }\end{array}$ \\
\hline \multirow[t]{2}{*}{3} & \multirow[t]{2}{*}{ BitMAC } & \multirow{2}{*}{$\begin{array}{l}\text { Centralized Scheduling TDMA } \\
\text { scheme }\end{array}$} & High Robustness & \multirow[t]{2}{*}{ Dissipate high energy } \\
\hline & & & Mitigates Collision & \\
\hline \multirow[t]{2}{*}{4} & \multirow[t]{2}{*}{ GMAC } & \multirow{2}{*}{$\begin{array}{l}\text { Centralized Scheduling TDMA } \\
\text { scheme }\end{array}$} & \multirow[t]{2}{*}{ High Energy Efficiency } & Large overheads \\
\hline & & & & Demands more gateway \\
\hline \multirow[t]{3}{*}{5} & \multirow[t]{3}{*}{ SMACS } & \multirow{3}{*}{$\begin{array}{l}\text { Distributed Scheduling with the } \\
\text { combination of TDMA and } \\
\text { FDMA or CDMA }\end{array}$} & Simple to Implement & High energy dissipation \\
\hline & & & \multirow[t]{2}{*}{ Mitigates Collision } & Difficult to find optimal route \\
\hline & & & & $\begin{array}{l}\text { Low degree of node } \\
\text { connectivity }\end{array}$ \\
\hline \multirow[t]{2}{*}{6} & \multirow[t]{2}{*}{ TRAMA } & \multirow[t]{2}{*}{$\begin{array}{l}\text { Localization based Scheduling+ } \\
\text { Global synchronization }\end{array}$} & Reduced Idle Listening & $\begin{array}{l}\text { Energy Dissipation due to } \\
\text { global synchronization }\end{array}$ \\
\hline & & & Reduces Overhearing & Complex to implement \\
\hline \multirow[t]{2}{*}{7} & \multirow[t]{2}{*}{$\mu \mathrm{MAC}$} & \multirow[t]{2}{*}{ Localization based Scheduling } & Reduced collision & \multirow[t]{2}{*}{ Dissipate High energy } \\
\hline & & & Simple to implement & \\
\hline \multirow[t]{2}{*}{8} & \multirow[t]{2}{*}{ EMMAC } & \multirow{2}{*}{$\begin{array}{l}\text { Localization based Scheduling } \\
\text { with TDMA scheme }\end{array}$} & Reduced collision & \multirow{2}{*}{$\begin{array}{l}\text { Active nodes are permanently } \\
\text { active hence it dissipate more } \\
\text { power }\end{array}$} \\
\hline & & & Simple to implement & \\
\hline \multirow[t]{2}{*}{9} & \multirow[t]{2}{*}{ PMAC } & \multirow[t]{2}{*}{$\begin{array}{l}\text { Localization based Scheduling } \\
\text { with TDMA scheme }\end{array}$} & Simple to implement & $\begin{array}{l}\text { Fixed size slot results in high } \\
\text { collision[10] }\end{array}$ \\
\hline & & & $\begin{array}{l}\text { Passive nodes improves } \\
\text { energy efficiency }\end{array}$ & $\begin{array}{l}\text { PMAC suffers from mobility } \\
\text { of nodes and Overheads[10] }\end{array}$ \\
\hline \multirow[t]{3}{*}{10} & \multirow[t]{3}{*}{ PACT } & \multirow[t]{3}{*}{$\begin{array}{l}\text { Rotating node roles with TDMA } \\
\text { scheme }\end{array}$} & \multirow[t]{3}{*}{ Enhances energy efficiency } & $\begin{array}{l}\text { Establishment of node slot } \\
\text { assignment effects energy } \\
\text { efficiency }\end{array}$ \\
\hline & & & & Complex method \\
\hline & & & & Increased collision \\
\hline 11 & BMA & Rotating node roles with TDMA & Enhances energy efficiency & Increased Idle Listening \\
\hline & & $\mathrm{sch}$ & & Increased collision \\
\hline 12 & MMAC & Handling Node Mobility & $\begin{array}{l}\text { Ensures } \quad \text { collision-free } \\
\text { scheduling }\end{array}$ & $\begin{array}{l}\text { Demand for position } \\
\text { information, which is } \\
\text { practically inconvenient and }\end{array}$ \\
\hline & & & $\begin{array}{l}\text { Dynamic adaptability over } \\
\text { the mobility patterns, hence } \\
\text { desirable to work in both } \\
\text { high and low sensor }\end{array}$ & dissipates excess energy \\
\hline
\end{tabular}




\begin{tabular}{|c|c|c|c|c|}
\hline & & & environments & \\
\hline \multirow[t]{2}{*}{13} & \multirow[t]{2}{*}{ FlexiMAC } & \multirow[t]{2}{*}{ Handling Node Mobility } & $\begin{array}{l}\text { Guaranteed end-to-end } \\
\text { delay for packet delivery } \\
\text { Better throughput }\end{array}$ & $\begin{array}{l}\text { Lacks robustness and } \\
\text { optimality }\end{array}$ \\
\hline & & & $\begin{array}{l}\text { Tolerable data access, and } \\
\text { vigorous self-healing }\end{array}$ & $\begin{array}{l}\text { Only parent-child and child- } \\
\text { parent communications are } \\
\text { optimal }\end{array}$ \\
\hline \multirow[t]{2}{*}{14} & \multirow[t]{2}{*}{ OMAC } & \multirow[t]{2}{*}{$\begin{array}{l}\text { TDMA based Receiver oriented } \\
\text { slot assignment }\end{array}$} & $\begin{array}{l}\text { Improves } \\
\text { Efficiency at the receiver } \\
\text { end }\end{array}$ & \multirow[t]{2}{*}{ High end-to-end Latency } \\
\hline & & & $\begin{array}{l}\text { Guaranteed collision } \\
\text { avoidance }\end{array}$ & \\
\hline \multirow[t]{2}{*}{15} & \multirow[t]{2}{*}{ Crankshaft } & \multirow[t]{2}{*}{$\begin{array}{l}\text { TDMA based Receiver oriented } \\
\text { slot assignment }\end{array}$} & $\begin{array}{l}\text { Improves } \\
\text { Efficiency at the receiver } \\
\text { end }\end{array}$ & \multirow[t]{2}{*}{$\begin{array}{l}\text { Unoccupied slots mitigates } \\
\text { overall throughput in low } \\
\text { traffic condition }\end{array}$} \\
\hline & & & $\begin{array}{l}\text { Guaranteed } \\
\text { avoidance }\end{array}$ & \\
\hline 16 & PicoRadio & $\begin{array}{l}\text { Utilizes } \\
\text { communication frequency with } \\
\text { CDMA scheme }\end{array}$ & $\begin{array}{l}\text { Energy efficient } \\
\text { Sender-based approach to } \\
\text { avoid collisions }\end{array}$ & High End-to-End Latency \\
\hline 17 & f-MAC & $\begin{array}{l}\text { FDMA based-Decomposes a } \\
\text { packet into several framelet }\end{array}$ & $\begin{array}{l}\text { Collisions avoidance } \\
\text { Minimizes interference }\end{array}$ & High energy dissipation \\
\hline \multirow[t]{2}{*}{18} & \multirow[t]{2}{*}{ LMAC } & \multirow[t]{2}{*}{ TDMA scheme } & $\begin{array}{l}\text { Minimizes } \\
\text { problems }\end{array}$ & \multirow[t]{2}{*}{$\begin{array}{l}\text { Node is able to transmit only } \\
\text { single message per frame }\end{array}$} \\
\hline & & & $\begin{array}{l}\text { Extend the network lifetime } \\
\text { by a factor } 2.4 \text { and } 3.8, \\
\text { compared to EMACs and } \\
\text { SMAC }\end{array}$ & \\
\hline \multirow[t]{2}{*}{19} & \multirow[t]{2}{*}{$\begin{array}{l}\text { Multichannel } \\
\text { LMAC }\end{array}$} & \multirow[t]{2}{*}{$\begin{array}{l}\text { TDMA scheme with multi- } \\
\text { channel support }\end{array}$} & $\begin{array}{l}\text { Only one half-duplex } \\
\text { transceiver per node is } \\
\text { sufficient }\end{array}$ & High energy consumption \\
\hline & & & $\begin{array}{l}\text { Collision avoidance, it } \\
\text { allows spatial re-use of slot }\end{array}$ & High End-to-End latency \\
\hline \multirow[t]{2}{*}{20} & \multirow[t]{2}{*}{ AI-LMAC } & \multirow[t]{2}{*}{$\begin{array}{l}\text { TDMA based, various } \\
\text { functionality protocol }\end{array}$} & Reduces latency & \multirow[t]{2}{*}{ Consumes more energy } \\
\hline & & & $\begin{array}{l}\text { Regulates the fairness issue } \\
\text { in a better way by } \\
\text { employing two-dimensional } \\
\text { fairness mechanism }\end{array}$ & \\
\hline \multirow[t]{2}{*}{21} & \multirow[t]{2}{*}{ MMSN } & \multirow[t]{2}{*}{$\begin{array}{l}\text { Utilizes different } \\
\text { communication frequency }\end{array}$} & Better Energy Efficiency & $\begin{array}{l}\text { Cannot guarantee collision- } \\
\text { free data transmission }\end{array}$ \\
\hline & & & & High control overhead \\
\hline \multirow[t]{2}{*}{22} & \multirow[t]{2}{*}{ Y-MAC } & \multirow[t]{2}{*}{$\begin{array}{l}\text { TDMA based synchronous } \\
\text { scheme }\end{array}$} & $\begin{array}{ll}\begin{array}{l}\text { Increases } \\
\text { throughput }\end{array} & \text { network } \\
\end{array}$ & \multirow[t]{2}{*}{$\begin{array}{l}\text { Energy wastage due to idle } \\
\text { listening and overhead }\end{array}$} \\
\hline & & & $\begin{array}{l}\text { Mitigates packet delivery } \\
\text { latency }\end{array}$ & \\
\hline \multirow[t]{2}{*}{23} & \multirow{2}{*}{$\begin{array}{l}\text { Practical } \\
\text { multichannel } \\
\text { MAC }\end{array}$} & \multirow{2}{*}{$\begin{array}{l}\text { Utilizes different } \\
\text { communication frequency }\end{array}$} & Increases throughput & \multirow[t]{2}{*}{ Dissipates more energy } \\
\hline & & & $\begin{array}{l}\text { This method elegantly } \\
\text { copes with narrowband } \\
\text { long-lasting interference }\end{array}$ & \\
\hline \multirow[t]{2}{*}{24} & \multirow[t]{2}{*}{ SS-TDMA } & $\begin{array}{l}\text { TDMA based, various } \\
\text { functionality protocol }\end{array}$ & $\begin{array}{l}\text { Better energy conservation } \\
\text { and clock synchronization }\end{array}$ & Exposed terminal problem \\
\hline & & & $\begin{array}{l}\text { An Efficient method to } \\
\text { handle issues: failed } \\
\text { sensors, sleeping sensors, } \\
\text { unidirectional links, and } \\
\text { unreliable links }\end{array}$ & Arbitrary clock drift \\
\hline
\end{tabular}




\begin{tabular}{|l|l|l|l|l|}
\hline 25 & RMAC & $\begin{array}{l}\text { TDMA based, various } \\
\text { functionality protocol }\end{array}$ & $\begin{array}{l}\text { It will estimate an optimal } \\
\text { number of mini-slots to be } \\
\text { used during the contention } \\
\text { period }\end{array}$ & $\begin{array}{l}\text { Large contention period } \\
\text { reduces collision but increases } \\
\text { per-packet latency }\end{array}$ \\
${$\cline { 4 - 5 }$} }$ \\
\end{tabular}

\section{CONCLUSIONS}

In this paper, a survey related to wireless sensor network's MAC layer protocols is presented. Initially, the implicit constraints of wireless medium and scarcity of available resources especially energy and memory are examined. Advancement in sensor network applications which instigated the rapidity of MAC development are identified. The main objective was to summarize the various traditional and schedule-based WSN MAC protocols, the basic technique of each protocol, its advantages, and drawbacks, which will lead to further understand the different MAC protocol designs needed in future. There are several issues in WSNs which are open for research: i. a sensor network with a complete package of throughput, energy consumption, delay, etc., with multi-hop nature which could encourage different application, has not been developed. Anyways an effort has been made in [40], but the outcome significantly differs from the ideal condition. ii. Till today no MAC protocol has been manifested as highly scalable or which could encourage auto-organization or configuration or healing. Hence, when a sensor network with thousands of nodes is deployed, it endures severe hurdle due to their one-hop radio neighborhood. iii. Demand for dynamic security in WSNs, which are generally not fulfilled by MAC protocols. Since energy efficiency is paramount in WSNs, MAC protocols should preserve energy by evading futile communication.

\section{REFERENCES:}

[1] Hiral B. Lad and Neha Parmar, "Contention Hybrid MAC Protocol for Wireless Sensor Networks," Int. J. Adv. Res. Comput. Sci. Softw. Eng., vol. 5, no. 6, pp. 579-582, 2015.

[2] Pei Huang, L. Xiao, and S. Soltani, "The Evolution of MAC Protocols in Wireless Sensor Networks : A Survey," IEEE Commun. Surv. TUTORIALS, vol. 15, no. 1, pp. 1-20, 2013.

[3] A. Bachir, L. Samper, D. Barthel, M. Heusse, and A. Duda, "Link cost and reliability of frame preamble MAC protocols," 2006 3rd Annu. IEEE Commun. Soc. Sens. Adhoc Commun. Networks, Secon 2006, vol. 2, no. C, pp. 632-638, 2007.

[4] Halil Yetgin, K. T. K. Cheung, M. El-Hajjar, and L. Hanzo, "Network-Lifetime Maximization of Wireless Sensor Networks," IEEE Access, vol. 3, pp. 2191-2226, 2015.

[5] R. Yadav, S. Varma, and N. Malaviya, "A survey of MAC protocols for wireless sensor networks," UbiCC J., vol. 4, no. 3, pp. 827$833,2009$.

[6] I. Demirkol, C. Ersoy, and F. Alagöz, "MAC Protocols for Wireless Sensor Networks: A Survey," IEEE Commun. Mag., vol. 44, no. November, pp. 115-121, 2006.

[7] K. Langendoen, "Medium Access Control in Wireless Networks," Nov. Sci. Publ., vol. 2, pp. 535-560, 2008.

[8] P. Karn, "MACA - A New Channel Access Method for Packet Radio," 9th ARRL Comput. Netw. Conf. London, Ontario, Canada, pp. $134-140,1990$.

[9] A. Woo and D. E. Culler, "A Transmission Control Scheme for Media Access in Sensor Networks," ACM Mobicom, Rome, Italy.

[10] A. Bachir, M. Dohler, T. Watteyne, I. Member, and I. S. Member, "MAC Essentials for Wireless Sensor Networks MAC Essentials for Wireless Sensor Networks," Commun. Surv. \{\&\} Tutorials, vol. 12, no. 2, pp. 222-248, 2010.

[11] S. Singh and C.S.Ragavendra, "PAMAS - power aware multi-access protocol with signaling for ad hoc networks," ACM SIGCOMM Comput. Commun. Rev., vol. 28, pp. 1048-1057, 1998.

[12] K. S. J. Pister and L. Doherty, “TSMP: Time Synchronized Mesh Protocol,” Proc. IASTED Int. Symp., pp. 391-398, 2008.

[13] J. Polastre, J. Hill, and D. Culler, "Versatile Low Power Media Access for Wireless Sensor Networks," SenSys '04 Proc. 2nd Int. Conf. Embed. networked Sens. Syst., pp. 95-107, 2004.

[14] M. Buettner, G. V. Yee, E. Anderson, and R. Han, "X-MAC: a short preamble MAC protocol for duty-cycled wireless sensor networks,” Proc. 4th Int. Conf. Embed. networked Sens. Syst. (SenSys 2006), pp. 307-320, 2006.

[15] L. Doherty, W. Lindsay, J. Simon, K. S. J. Pister, and H. Ave, "Channel-specific wireless sensor network path analysis," 16th Int. Conf. Comput. Commun. Networks, 2007 (ICCCN '07), pp. 89-94, 2007.

[16] S. Coleri and P. Varaiya, "PEDAMACS : Power Efficient and Delay Aware Medium Access Protocol for Sensor Networks," IEEE Trans. Mob. Comput., vol. 5, no, pp. 920-930, 2006.

[17] M. Ringwald and K. Römer, "BitMAC: A Deterministic, Collision-Free, and Robust MAC Protocol for Sensor Networks," Proc. Second IEEE Eur. Work. Wirel. Sens. Networks Appl. (EWSN 2005), pp. 1-13, 2005.

[18] M. I. Brownfield, K. Mehrjoo, A. S. Fayez, and N. J. Davis, "Wireless sensor network energy-adaptive mac protocol," Proceedings 3rd IEEE Consum. Commun. Netw. Conf. 2006., pp. 778-782, 2005.

[19] K. Sohrabi, J. Gao, V. Ailawadhi, and G. J. Pottie, "Protocols for Self-Organisation of a Wireless Sensor Network," IEEE Pers. Commun., no. September 1999, pp. 16-27, 2000.

[20] V. Rajendran, K. Obraczka, and J. J. Garcia-Luna-Aceves, "Energy-Efficient, Collision-Free Medium Access Control for Wireless Sensor Networks," SenSys. Los Angeles, CA, USA ACM, pp. 445-452, 2003.

[21] A. Barroso, U. Roedig, and C. Sreenan, "uMAC: An Energy-Efficient Medium Access Control for Wireless Sensor Networks," EWSN. IEEE, pp. 445-452, 2005.

[22] L. van H. and P. Havinga, “'Advantages of a TDMA based, energy- efficient, self-organizing MAC protocol for WSNs, ” VTC, pp. $1598-1602,2004$.

[23] P. Cheong and I. Oppermann, “An Energy-Efficient Positioning-Enabled MAC Protocol (PMAC) for UWB Sensor Networks," IST Mob. Wirel. Commun. Summit, pp. 95-107, 2005.

[24] C. Chien, G. Pei, C. Chien, R. S. Co, and T. Oaks, "Low power TDMA in large wireless sensor networks," IEEE Mil. Commun. Conf. 2001. MILCOM 2001. Commun. Network-Centric Oper. Creat. Inf. Force, vol. 1, no. c, pp. 347-351, 2001.

[25] J. Li and G. Y. Lazarou, "A bit-map-assisted energy-efficient MAC scheme for wireless sensor networks," Proc. third Int. Symp. Inf. Process. Sens. networks - IPSN’04, pp. 55-60, 2005. 
[26] Zartash Afzal Wzmi, M. Ali, and T. Suleman, "MMAC: A Mobility-Adaptive, Collision-Free MAC Protocol for Wireless Sensor Networks," IPCCC. IEEE, pp. 401-407, 2005.

[27] R. Cardell-Oliver, W. L. Lee, and A. Datta, "FlexiMAC: A flexible TDMA-based MAC protocol for fault-tolerant and energy-efficient wireless sensor networks," IEEE Int. Conf. Networks, ICON, vol. 2, pp. 337-342, 2006.

[28] C. Hui, K. W. Parker, and A. Arora, "O-MAC: A receiver centric power management protocol," Proc. - Int. Conf. Netw. Protoc. ICNP, pp. 311-320, 2006.

[29] G. P. Halkes and K. G. Langendoen, "Crankshaft: An Energy-Efficient MAC-Protocol for Dense Wireless Sensor Networks," Springer-Verlag Berlin Heidelb., pp. 228-244, 2007.

[30] J. M. Rabaey, M. J. Ammer, J. L. da Silva, D. Patel, and S. Roundy, "Picoradio supports ad hoc ultra-low power wireless networking," IEEE Comput., vol. 33, pp. 42-48, 2000.

[31] U. Roedig, A. Barroso, and C. J. Sreenan, "f-MAC : A Deterministic Media Access Control Protocol Without Time Synchronization," pp. 276-291, 2006.

[32] L. van H. and P. Havinga, "A lightweight medium access protocol (LMAC) for wireless sensor networks: Reducing Preamble Transmis- sions and Transceiver State Switches," Int. Conf. Networked Sens. Syst., 2004.

[33] O. Incel, S. Dulman, and P. Jansen, "Multi-channel Support for Dense Wireless Sensor Networking," Smart Sens. Context, vol. 4272, pp. $1-14,2006$

[34] S. Chatterjea, L.F.W. van Hoesel, and P.J.M. Havinga, "AI-LMAC: An Adaptive, Information-Centric and Lightweight MAC Protocol for Wireless Sensor Networks,” Proc. 2004 Intell. Sensors, Sens. Networks Inf. Process. Conf. 2004., pp. 381-388, 2004.

[35] G. Zhou et al., "A multifrequency MAC specially designed for wireless sensor network applications," ACM Trans. Embed. Comput. Syst., vol. 9, no. 4, pp. 1-41, 2010.

[36] Y. Kim, H. Shin, and H. Cha, "Y-MAC: An energy-efficient multi-channel MAC protocol for dense wireless sensor networks," Proc. 2008 Int. Conf. Inf. Process. Sens. Networks, IPSN 2008, pp. 53-63, 2008.

[37] H. K. Le, D. Henriksson, and T. Abdelzaher, "A Practical Multi-channel Media Access Control Protocol for Wireless Sensor Networks," Int. Conf. Inf. Process. Sens. Networks, pp. 70-81, 2008

[38] M. Arumugam and S. Kulkarni, "SS-TDMA: A SELF-STABILIZING MAC FOR SENSOR NETWORKS," Sens. Netw. Oper. Wiley-IEEE Press, pp. 1-32, 2006.

[39] S. Datta, "RMAC: a randomized adaptive access control algorithm for sensor networks," SANPA, Boston, MA, pp. 1-11, 2006.

[40] K. Voulgaris, I. Ashraf, T. Karveli, and A. H. Aghvami, "Energy efficiency of p-persistent CSMA under variable traffic conditions," IEEE Int. Symp. Pers. Indoor Mob. Radio Commun. PIMRC, no. June, 2008. 